\title{
Selecting dominants and subordinates at conflict outcome can confound the effects of prior dominance or subordination experience
}

\author{
Jean Bégin a, Jacques P. Beaugrand $\mathbf{a}^{*}$ and René Zayan $b$ \\ a Département de Psychologie, Université du Québec à Montréal, \\ C.P. 8888, suc. A, Montréal, Québec, H3C 3 P8 (Canada) \\ b Faculté de Psychologie, Université de Louvain, \\ B-1348 Louvain-la-Neuve, Belgique \\ Footnote *) Corresponding author. \\ Email: beaugrand.jacques@uqam.ca
}

$\{$ BP1051\}

The present text is a preprint and is not exactly conform to that which appeared in Behavioural Processes, 36, (1996), 219-226.

\section{Abstract}

Individuals with a previous experience of dominance are likely to be dominants in further encounters. To test this effect, individuals with a previous experience of dominance are used for the experiments. One way to obtain such individuals is to let opponents «self-select»: encounters between pairs of more or less equivalent opponents are staged and one selects ex post facto the dominant and subordinate from the ensuing conflict. This paper formally shows that the selection of dominant and subordinate animals modifies the dominance probability functions of the two corresponding sub-samples of animals. As a result, the propensity of previous winners to win again and of previous losers to repeat their loss can be attributed to this artefact rather than to prior social experience. A simulation confirms the results of the theoretical analysis. This result has serious methodological implications. When one relies solely on selection to obtain winners and losers, equiprobability is no longer the appropriate null hypothesis against which prior social experience effects have to be tested. To clearly demonstrate the effect of dominance experience, one must show that prior winners defeat neutral opponents in at least of all cases; reciprocally, to show that prior subordinate experience plays a role, prior losers must win in less than of all fights against neutral opponents; finally, to conclude that a combined effect of the two kinds of prior experience is in operation, one must obtain that prior winners defeat prior losers in more than $83 \%$ of all planned conflicts. The present result does not imply that experience effects are not at work when the selection procedure is used, but that the procedure used to show their effects is inadequate because effects of experience on a subsequent encounter are confounded with those introduced by statistical selection.

Key-words: Dominance; Individual differences; Self-selected dominants; Prior-experience; Mathematical proof.

\section{Introduction}

Dominance is now admitted to be affected by factors related to the history of two given contestants. Among 
experiential factors, prior dominance experience appears to account for the increase in propensity to dominate again, while recent subordination experience seems to produce the counter effect (Francis, 1983; Beaugrand and Zayan, 1985; Beacham and Newman, 1987). Usually, there are two ways of obtaining dominants and subordinates to test for the effect of recent experience upon ulterior dominance. One is to «impose» such experience on the contestant by deciding a priori (and randomly) which individual will receive a dominance experience and which will receive a subordination experience. There is a variety of ways to manipulate dominance or subordination, e.g., by using size and prior-residency asymmetries. For instance, if a large resident hen is forced to receive a smaller intruder in its home cage, the probability is great that the former will defeat the latter. The second way to obtain dominants is to stage encounters between pairs of equivalent opponents and to select ex post facto the dominant and subordinate from the ensuing conflict. This type of selection follows a more «natural» method for the division of winners and losers than the imposing of prior experience. However, this selection of winners and losers poses serious methodological limits precluding the drawing of a straightforward inference about the precise role of social experience in determining dominance and, eventually, rank order in a hierarchy. Some unspecified individual attribute such as fighting ability, or hormonal level, may account for the fact that the winner in a pre-experimental encounter is also more likely to dominate during a second encounter. The aim of the present theoretical paper is to formally examine the consequences of selecting dominant and subordinate animals either a priori, vs ex post facto.

The experimental demonstration of prior experience effects is usually carried out in the following manner. An initial encounter $\left(\mathrm{I}_{\mathrm{in}}\right)$ is planned between two individuals unfamiliar with one another; a dominance relation (noted) is established at the issue of each of these conflicts between the pair-members: A (the dominants, by convention) and B (the subordinates). Following this, two further conflicts $\left(\mathrm{I}_{1}\right.$ and $\left.\mathrm{I}_{2}\right)$ can be made between these two protagonists and a third one, called $\mathrm{C}$, which is considered «neutral» since it has not recently met any animal. It is also possible to arrange a conflict $\mathrm{I}_{3}$ between an $\mathrm{A}$ and a $\mathrm{B}$ which have not previously met, i.e., obtained from two different $\mathrm{I}_{\mathrm{in}}$. To indicate their unfamiliarity with each other, the notation $\mathrm{A}$ ! and $\mathrm{B}$ ! will be used.

To summarize, we have an initial confrontation

$$
I_{\text {in }}=A \text { us } B \quad \wedge \quad A>B \text { (by definition) }
$$

which creates one of the following three possibilities

$$
\begin{gathered}
I_{1}=A \text { us } C \wedge(A>C \vee C>A) \\
I_{2}=B \text { us } C \wedge(B>C \vee C>B) \\
I_{3} \doteq A^{\prime} \text { us } B^{\prime} \wedge\left(A^{\prime}>B^{\prime} \vee B^{\prime}>A\right)
\end{gathered}
$$

Assuming that the three types of unfamiliar animals A (or $\mathrm{A} !), \mathrm{B}$ (or $\mathrm{B} !$ ) and $\mathrm{C}$ have an equivalent propensity to dominate, the outcome of these three conflict types should be equiprobable. However, empirical data (e.g., Beaugrand and Zayan, 1985) show that it is not the case: the probability of type A dominating type $C$ at $I_{1}$ is greater than 0.5 , which supports the hypothesis for the existence of prior experience of dominance in type A. 
Similarly, the probability of type $\mathrm{B}$ dominating $\mathrm{C}$ in $\mathrm{I}_{2}$ is lower than 0.5 , which supports the hypothesis that prior experience of subordination is at work in type B. Finally, the combined effect of prior dominance experience in $\mathrm{A}$ ! and prior subordination experience in $\mathrm{B}$ ! would explain the high proportion of gains by $\mathrm{A}$ ! over $\mathrm{B}$ !; as expected, $\mathrm{A}$ ! more frequently win against a $\mathrm{B}$ ! opponent than against a $\mathrm{C}$ opponent, and $\mathrm{B}$ ! less frequently win against $A$ ! than against $C$ (Beaugrand and Zayan, 1985). However, the preceding reasoning unrealistically assumes the equiprobability of gains for any two pair members. In other words, one postulates that individuals A, B and C are equivalent right from the start, i.e., either that (a) no individual characteristic plays a determining role upon conflict outcome, or that (b) such characteristics exist, but equally for all three protagonists.

However, postulate (b) is only plausible if many pre-experimental controls/matchings are carried out, thus limiting the generality of the explanation. In so far as postulate (a) is concerned, inferring that a future dominant at $\mathrm{I}_{\text {in }}$ does not possess any advantageous characteristic over its future subordinate is clearly unrealistic. For instance, differences among the individuals may exist but recent agonistic experiences could over-shadow them. One could ask why one animal won in the first place. Rather, it would seem that some intrinsic (or dispositional) factors, which do not depend upon the sequential order of encounters, might be considered to determine dominance.

\section{P1. Dispositional factors which are allowed to be self-selected are sufficient to account for the disproportionate gains which have been, up to now, attributed to the effect of prior dominance/subordination experience.}

In order to examine proposition P1, one must suppose that there exists such a dispositional factor for dominance, i.e., a factor proper to each animal and which directly determines conflict outcome between two unacquainted protagonists. In other words, if one animal has a greater predisposition to win than another, it will win on its first encounter. Several characteristics of the animal can contribute to its disposition for dominance, but they must combine to form some kind of composite factor which corresponds to the construct we have called «dominance factor». It is realistic to assume that this factor is distributed in a continuous fashion throughout the concerned population, be it homogeneous or not. In addition to this, it is possible, and even desirable, to suppose that this factor is stable in time, or at least within the time interval required for the establishment of the dominance structure. Otherwise, it would become impossible to predict outcome and to develop the present approach.

For a given population, numerical values $x$ of the «dominance factor» will be used to define a probability function

$$
F(X \leq x)=F(x)
$$

which indicates the probability of gain of individual $S$ possessing a value $x$ on the factor of dominance when $S$ is compared to its own population. This function shows the properties of any probabilistic function; it is continuous, non-decreasing, and possesses a derived probability density function $\mathrm{f}(x)$.

What is important to realize here is that being initially labelled as dominant (A) or subordinate (B) at the outcome of $\mathrm{I}_{\mathrm{in}}$, modifies the dominance probability functions corresponding to each of the newly formed samples. The probability of an individual of value $x$ being classed as an $\mathrm{A}$ at $\mathrm{I}_{\mathrm{in}}$ is thus given by 


$$
F(A \mid x)=P(X \leq x)=\mathrm{F}(x)
$$

where $\mathrm{P}(\mathrm{A} \mid x)$ stands for the conditional probability that an individual is classified in set A given that it has dominance value $x$. The probability of $x$ being classed $\mathrm{B}$ at $\mathrm{I}_{\text {in }}$ is

$$
P(B \mid x)=P(X>x)=1-F(x)
$$

Consequently, the probability density function for each sample becomes, respectively,

$$
\mathrm{a}(x)=2 \mathrm{f}(x) \mathrm{F}(x)
$$

for dominants, and

$$
\mathrm{b}(x)=2 \mathrm{f}(x)(1-\mathrm{F}(x))=2 \mathrm{f}(x)-\mathrm{a}(x)
$$

for subordinates. Equations (6) and (7) can be both derived from Bayes' theorem:

$$
f(A \mid x)=\alpha(x)=\frac{f(x) P(A \mid x)}{\int f(x) P(A \mid x) d x}
$$

Using equation (2), it now becomes possible to calculate the winning probability of each protagonist for conflicts $\mathrm{I}_{1}, \mathrm{I}_{2}$ and $\mathrm{I}_{3}$. First, note that for any animal $\mathrm{C}, \mathrm{c}(x)=\mathrm{f}(x)$. The probability that A dominates a given $\mathrm{C}$ at $\mathrm{I}_{1}$ will thus be given by 


$$
\begin{aligned}
P\left(x_{C}<x_{A}\right) & =\int_{-\infty}^{+\infty}\left(a(x) \int_{-\infty}^{x} \mathrm{f}(t) d t\right) d x \\
& =\int_{-\infty}^{+\infty} 2 \mathrm{f}(x) \mathrm{F}(x) \mathrm{F}(x) d x \\
& =2 \int_{-\infty}^{+\infty} \mathrm{f}(x)(\mathrm{F}(x))^{2} d x \\
& =2 \frac{1}{1+2} \\
& =\frac{2}{3}
\end{aligned}
$$

The transformation in (9) marked by an * was made possible by a property explained in the appendix.

P2. The probability that a self-selected winner will defeat a self-selected loser to which it is unacquainted is of 5/6.

The probability that $B$ will dominate $C$ at $I_{2}$, is given by (10). That $A !$ will dominate $B !$ at $I_{3}$ is given by (11).

$$
\begin{aligned}
P\left(x_{C}<x_{B}\right) & =\int_{-\infty x}^{+\infty}\left(\mathrm{b}(x) \int_{-\infty}^{x} \mathrm{f}(t) d t\right) d x \\
& =\int_{-\infty}^{+\infty} 2 \mathrm{f}(x)(1-\mathrm{F}(x) \mathrm{F}(x) d x \\
& =2 \int_{-\infty}^{+\infty} \mathrm{f}(x) \mathrm{F}(x) d x-2 \int_{-\infty}^{+\infty} \mathrm{f}(x)(\mathrm{F}(x))^{2} d x \\
& =2 \frac{1}{1+1}-2 \cdot \frac{1}{2+1} \\
& =\frac{1}{3}
\end{aligned}
$$




$$
\begin{aligned}
P\left(x_{B}<x_{A}\right) & =\int_{-\infty}^{+\infty}\left(\begin{array}{c}
x \\
\left.\mathrm{a}(x) \int_{-\infty} \mathrm{b}(t) d t\right) d x
\end{array}\right. \\
& =\int_{-\infty}^{+\infty}\left(2 \mathrm{f}(x) \mathrm{F}(x) \int_{-\infty}^{x}(2 \mathrm{f}(t)(1-\mathrm{F}(t))) d t\right) d x \\
& =\int_{-\infty}^{+\infty}\left(2 \mathrm{f}(x) \mathrm{F}(x)\left(\int_{-\infty}^{x} 2 \mathrm{f}(t) d t-\int_{-\infty}^{x} 2 \mathrm{f}(t) \mathrm{F}(t) d t\right)\right) d x \\
& =\int_{-\infty}^{+\infty}\left(2 \mathrm{f}(x) \mathrm{F}(x)\left(2 \mathrm{~F}(x)-2 \frac{(\mathrm{F}(x))^{2}}{2}\right)\right) d x \\
& =\int_{-\infty}^{+\infty}\left(4 \mathrm{f}(x)(\mathrm{F}(x))^{2}\right) d x-\int_{-\infty}^{+\infty}\left(2 \mathrm{f}(x)(\mathrm{F}(x))^{3}\right) d x \\
& =4 \frac{1}{2+1}-2 \frac{1}{3+1} \\
& =\frac{5}{6}
\end{aligned}
$$

Due to the general nature of the functions used, these conclusions are totally independent of any form resulting in a distribution of the dominance factor, which can be normal, uniform, exponential, etc. In order to illustrate the preceding analysis, a normal $N(0,1)$ distribution was chosen. Fig. 1 illustrates the functions $\mathrm{a}(x), \mathrm{b}(x)$ and $\mathrm{c}(x)(=\mathrm{f}(x))$ at $\mathrm{I}_{\mathrm{in}}$. It is important to note that functions $\mathrm{a}(x)$ and $\mathrm{b}(x)$, obtained after dividing samples into winners and losers, are not normal distributions, although they are mirror images of each other. 


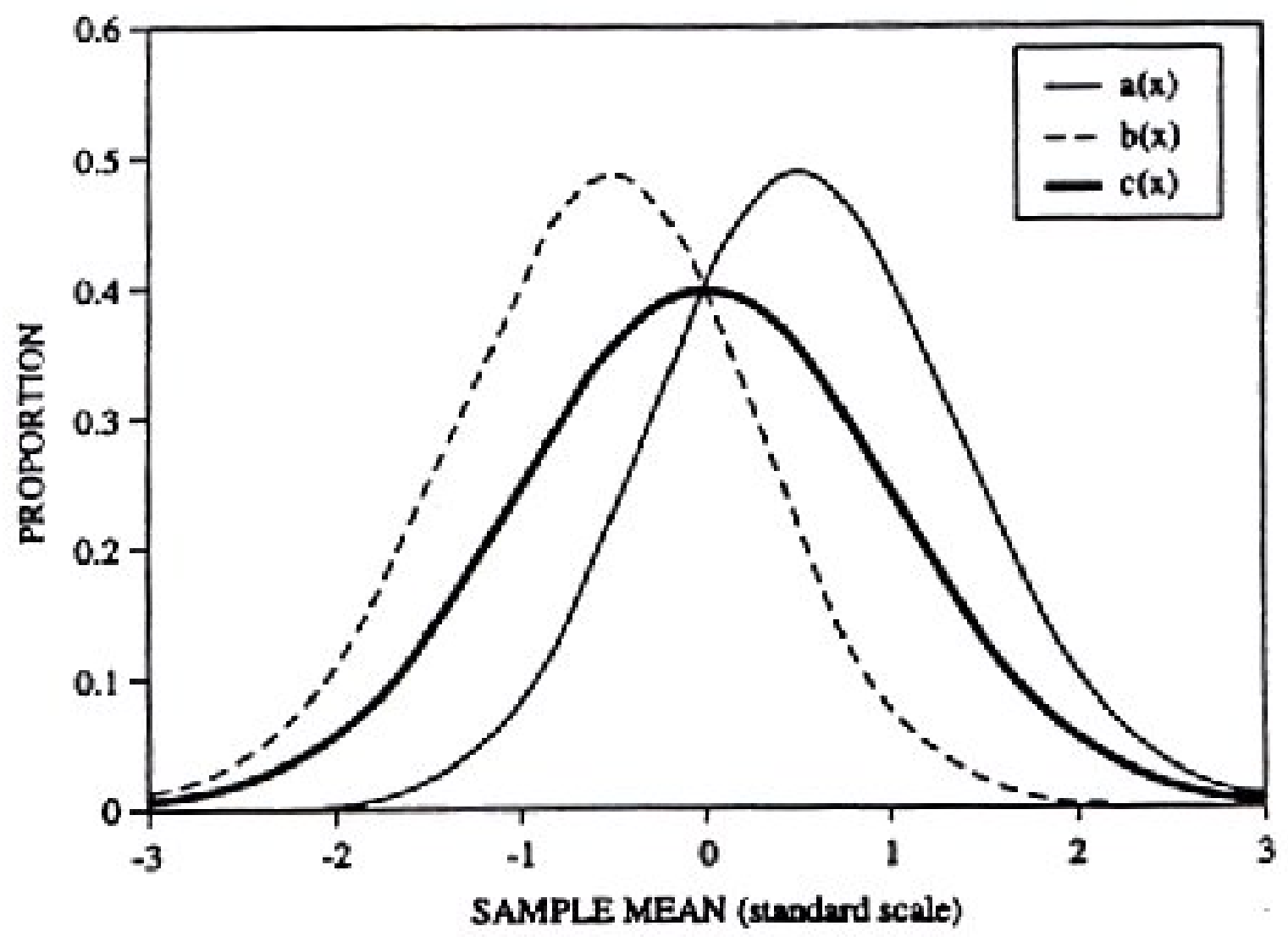

Figure 1. Illustration of the effect of dominant and subordinate selection. A normal $N(0,1)$ distribution is used.

Function $\mathrm{c}(x)$ corresponds to the original distribution $(=\mathrm{f}(x))$. Functions $\mathrm{a}(x)$ and $\mathrm{b}(x)$ were obtained after the division of winners and losers into their respective sub-samples.

\section{Discussion}

The above demonstration has serious methodological implications. When one relies solely on ex post facto «selection» to obtain winners and losers, equiprobability is no longer the appropriate null hypothesis against which experience effects have to be tested. To clearly demonstrate the effect of dominance experience one must show that individuals of type $\mathrm{A}$ defeat $\mathrm{C}$ opponents significantly in at least of all cases at $\mathrm{I}_{1}$; reciprocally, to show that prior subordinate experience plays a role, individuals of type B must win in less than of all fights against type $\mathrm{C}$ at $\mathrm{I}_{2}$; finally, to conclude that a combined effect of the two kinds of prior experience is in operation, one must obtain that type $A$ ! defeats type $B$ ! in more than $5 / 6$ of all planned conflicts at $\mathrm{I}_{3}$. This means that the results of several papers e.g., Frey and Miller (1972), Beaugrand and Zayan (1985), Franck and Ribowski (1988), Beaugrand et al. (1991) to name a few, must be interpreted cautiously. Since these studies relied upon selection to obtain dominants and subordinates, the effects that had once been attributed to prior experience can now be re-interpreted in terms of statistical selection.

Examination of the results of various research which relied upon selection indicates that none of them satisfy the aforementioned criteria. One exception must be made for Zayan (1987) who actually obtained more than $5 / 6$ (100\% in some cases) in successive asymmetric pairings of unfamiliar hens.

The present analysis does not set out to cast doubt on existing evidence on the effects of prior experience in cases where the methodology used was to impose experience a priori on individuals (e.g., Francis, 1983). It 
does argue, however, that when a selection procedure is used, effects of experience on a subsequent encounter are confounded with those introduced by statistical selection. Such results cannot serve as evidence for prior experience effects, unless they significantly pass the criteria specified by equations (9), (10) and (11). This does not imply that experience effects are not at work when the selection procedure is used, for in reality they most probably are, but it is important to note that the procedure used to show their effects is inadequate.

This methodological conclusion concerns mainly the experiments which we design in order to gain more understanding on the mechanism of dominance hierarchy formation. But, it also has paradoxical implications in our understanding of naturally forming hierarchies because potential participants in genuine social hierarchies sort themselves out naturally and so doing probably «self-select» each other. They do not necessarily control past histories of their opponents through rigged contests as we do in our experiments. This emphasizes the underlying methodological difficulty, or even impossibility, of proving that prior experience plays a role in the natural process of hierarchy formation in animals. Such a role can only be inferred from evidence obtained in experimentally controlled, but highly unnatural, situations.

\section{Acknowledgments}

This work was supported by a NSERC operating grant to the second author.

\section{Appendix}

Equation (9) was made possible by the property

$$
\int_{-\infty}^{+\infty}\left(\mathrm{f}(x)(\mathrm{F}(x))^{M}\right) d x=\frac{1}{N+1} \quad N \geq 0
$$

as demonstrated as follows. Given equation (5), the corresponding cumulative function of density function $\mathrm{f}(x)$, then 


$$
\begin{aligned}
& \text { if } \mathrm{F}(x)=u \text {, then } \mathrm{f}(x) d x=d u \text { where } \\
& \begin{aligned}
\int_{-\infty}^{t}\left(\mathrm{f}(x)(\mathrm{F}(x))^{M}\right) d x & =\int_{-\infty}^{t} u^{N} d u \\
& =\left.\frac{u^{N+1}}{N+1}\right|_{-\infty} ^{t} \\
& =\left.\frac{(\mathrm{F}(x))^{N+1}}{N+1}\right|_{-\infty} ^{t} \\
& =\frac{(\mathrm{F}(t))^{N+1}}{N+1}-\frac{(\mathrm{F}(-\infty))^{N+1}}{N+1} \\
& =\frac{(\mathrm{F}(t))^{N+1}}{N+1}-\frac{0^{N+1}}{N+1} \\
& =\frac{(\mathrm{F}(t))^{N+1}}{N+1}
\end{aligned}
\end{aligned}
$$

and, in particular,

$$
\begin{aligned}
\int_{-\infty}^{+\infty}\left(\mathrm{f}(x)(\mathrm{F}(x))^{M}\right) d x & =\frac{(\mathrm{F}(+\infty))^{N+1}}{N+1} \\
& =\frac{1^{N+1}}{N+1} \\
& =\frac{1}{N+1}
\end{aligned}
$$

\section{References}

Beacham, J. L. and Newman, J.A., 1987. Social experience and the formation of dominance relationships in the pumpkinseed sunfish (Lepomis gibbosus). Anim. Behav., 35: 1560-1563.

Beaugrand, J., Goulet, C. and Payette, D., 1991. Outcome of dyadic conflict in male green swordtail fish, Xiphophorus helleri: effects of body size and prior dominance. Anim. Behav., 41: 417-424.

Beaugrand, J.P. and Zayan, R., 1985. An experimental model of aggressive dominance in Xiphophorus helleri (Pisces, Poeciliidae). Behav. Proc., 10: 1-52. 
Francis, R.C., 1983. Experiential effects on agonistic behavior in the paradise fish, Macropodus opercularis. Behaviour, 85: 292-313.

Franck, D. and Ribowski, A., 1988. Influences of prior agonistic experiences on aggression measures in the male swordtail (Xiphophorus helleri). Behaviour, 103: 217-239.

Frey, D.F. and Miller, R.J., 1972. The establishment of dominance relationships in the blue gourami, Trichogaster trichopterus (Pallas). Behaviour, 42: 8-62.

Zayan, R., 1987. Recognition between individuals indicated by aggression and dominance in pairs of domestic fowl. In: R. Zayan and I.J.H. Duncan (Editors), Cognitive aspects of social behaviour in the domestic fowl, pp. 321-438, Elsevier, Amsterdam. 